\title{
MAXIMUM HYPERGRAPHS WITHOUT REGULAR SUBGRAPHS
}

\author{
JAEHOON KIM ${ }^{1}$ \\ Department of Mathematics, University of Illinois \\ Urbana, IL, 61801, USA \\ e-mail: kim805@illinois.edu \\ AND \\ Alexandr V. KostochKA ${ }^{2}$ \\ University of Illinois at Urbana-Champaign, \\ Urbana, IL 61801, USA \\ Sobolev Institute of Mathematics \\ Novosibirsk 630090, Russia \\ e-mail: kostochk@math.uiuc.edu
}

\begin{abstract}
We show that an $n$-vertex hypergraph with no $r$-regular subgraphs has at most $2^{n-1}+r-2$ edges. We conjecture that if $n>r$, then every $n$-vertex hypergraph with no $r$-regular subgraphs having the maximum number of edges contains a full star, that is, $2^{n-1}$ distinct edges containing a given vertex. We prove this conjecture for $n \geq 425$. The condition that $n>r$ cannot be weakened.
\end{abstract}

Keywords: hypergraphs, set system, subgraph, regular graph.

2010 Mathematics Subject Classification: 05C65.

\section{REFERENCES}

[1] N. Alon, S. Friedland, and G. Kalai, Regular subgraphs of almost regular graphs, J. Combin. Theory (B) 37 (1984) 79-91. doi:10.1016/0095-8956(84)90047-9

\footnotetext{
${ }^{1}$ Research of this author is partially supported by the Arnold O. Beckman Research Award of the University of Illinois at Urbana-Champaign.

${ }^{2}$ Research of this author is supported in part by NSF grant DMS-0965587 and by the Ministry of education and science of the Russian Federation (Contract no. 14.740.11.0868).
} 
[2] M. Kano, Regular subgraphs of a regular graph, Annals of the New York Academy of Sciences 576 (1989) 281-284.

doi:10.1111/j.1749-6632.1989.tb16409.x

[3] D. Mubayi and J. Verstraëte, Two-regular subgraphs of hypergraphs, J. Combin. Theory (B) 99 (2009) 643-655.

doi:10.1016/j.jctb.2008.10.005

[4] L. Pyber, Regular subgraphs of dense graphs, Combinatorica 5 (1985) 347-349. doi:10.1007/BF02579250

[5] L. Pyber, V. Rödl, and E. Szemerédi, Dense graphs without 3-regular subgraphs, J. Combin. Theory (B) 63 (1995) 41-54.

doi:10.1006/jctb.1995.1004

[6] V. Rödl and B. Wysocka, Note on regular subgraphs, J. Graph Theory 24 (1997) $139-154$. doi:10.1002/(SICI)1097-0118(199702)24:2〈139::AID-JGT2 33.0.CO;2-R

[7] V.A. Tashkinov, 3-regular subgraphs of 4-regular graphs, Mat. Zametki 36 (1984) $239-259$.

[8] V.A. Tashkinov, Regular parts of regular pseudographs, Mat. Zametki 43 (1988)) $263-275$.

Received 20 June 2012

Revised 30 January 2013

Accepted 31 January 2013 